\title{
Computationally efficient flux variability analysis
}

\author{
Steinn Gudmundsson ${ }^{1}$, Ines Thiele $e^{1,2^{*}}$
}

\begin{abstract}
Background: Flux variability analysis is often used to determine robustness of metabolic models in various simulation conditions. However, its use has been somehow limited by the long computation time compared to other constraint-based modeling methods.

Results: We present an open source implementation of flux variability analysis called fastFVA. This efficient implementation makes large-scale flux variability analysis feasible and tractable allowing more complex biological questions regarding network flexibility and robustness to be addressed.

Conclusions: Networks involving thousands of biochemical reactions can be analyzed within seconds, greatly expanding the utility of flux variability analysis in systems biology.
\end{abstract}

\section{Background}

Flux balance analysis (FBA) $[1,2]$ is concerned with the following linear program (LP)

$$
\begin{array}{ll}
\max _{\mathbf{v}} & \mathbf{c}^{T} \mathbf{v} \\
\text { subject to } & \mathbf{S v}=\mathbf{0} \\
& \mathbf{v}_{l} \leq \mathbf{v} \leq \mathbf{v}_{u}
\end{array}
$$

where the matrix $\mathbf{S}$ is an $m \times n$ stoichiometry matrix with $m$ metabolites and $n$ reactions and $\mathbf{c}$ is the vector representing the linear objective function. The decision variables $\mathbf{v}$ represent fluxes, with $\mathbf{V} \subseteq \mathbb{R}^{n}$ and vectors $\mathbf{v}_{l}$ and $\mathbf{v}_{u}$ specify lower and upper bounds, respectively. The constraints $\mathbf{S v}=\mathbf{0}$ together with the upper and lower bounds specify the feasible region of the problem.

Flux variability analysis (FVA) [3] is used to find the minimum and maximum flux for reactions in the network while maintaining some state of the network, e.g., supporting $90 \%$ of maximal possible biomass production rate.

Applications of FVA for molecular systems biology include, but are not limited to, the exploration of alternative optima of (1) [3], studying flux distributions under suboptimal growth [4], investigating network flexibility and network redundancy [5], optimization of process feed formulation for antibiotic production [6],

* Correspondence: ithiele@hi.is

${ }^{1}$ Center for Systems Biology, University of Iceland, Reykjavik, Iceland

Full list of author information is available at the end of the article and optimal strain design procedures as a pre-processing step $[7,8]$.

Let $\mathbf{w}$ represent some biological objective such as biomass or ATP production. After solving (1) with $\mathbf{c}=\mathbf{w}$, FVA solves two optimization problems for each flux $v_{i}$ of interest

$$
\begin{array}{ll}
\max _{\mathbf{v}} / \min _{\mathbf{v}} & v_{i} \\
\text { subject to } & \mathbf{S v}=\mathbf{0} \\
& \mathbf{w}^{T} \mathbf{v} \geq \ddagger Z_{0} \\
& \mathbf{v}_{\boldsymbol{l}} \leq \mathbf{v} \leq \mathbf{v}_{\boldsymbol{u}}
\end{array}
$$

where $Z_{0}=\mathbf{w}^{T} \mathbf{v}_{0}$ is an optimal solution to (1), $\gamma$ is a parameter, which controls whether the analysis is done w.r.t. suboptimal network states $(0 \leq \gamma<1)$ or to the optimal state $(\gamma=1)$. Assuming that all $n$ reactions are of interest, FVA requires the solution of $2 n$ LPs. While FVA is clearly an embarrassingly parallel problem and is therefore ideally suited for computer clusters, this note focuses on how FVA can be run efficiently on a single CPU. A multi-CPU implementation of fastFVA can be done in the same way as for FVA, i.e., by distributing subsets of the $n$ reactions to individual CPUs. It is expected to give almost linear speedup for sufficiently large problems.

\section{Implementation}

A direct implementation of FVA iterates through all the $n$ reactions and solves the two optimization problems in (2) from scratch each time by calling a specialized LP 
solver, such as GLPK [9] or CPLEX (IBM Inc.) At iteration $i, i=1,2, \ldots, n$ all elements of $\mathbf{c}$ are zero except $c_{i}$ $=1$. Since the only difference between each iteration is a change in the objective function, i.e., the feasible region does not change, solving the LPs from scratch is wasteful. Each time a LP is solved, the solver has to spend some effort in finding a feasible solution. Once a feasible solution is found, the solver then proceeds to locate the optimum. The small changes in the objective function suggest that, on average, the optimum for iteration $i$ does not lie far away from the optimum for iteration $i+1$. With Simplex-type LP algorithms, this property can be exploited by solving problem (1) from scratch and then solving the subsequent $2 n$ problems of (2) by starting from the last optimum solution each time (warm-starts). It should be noted that the default behavior of some Simplex-type solvers is to use warmstarts when a sequence of LPs is solved within the same application call. However, current implementations of FVA do not make use of this option (c.f. [10]). Furthermore, for increased efficiency, model preprocessing (presolving) should be disabled after solving the initial problem $P$. Given a value of $0<\gamma \leq 1$, fastFVA performs the following procedure

Setup problem (1), denote it by $P$

Solve $P$ from scratch to get $\mathbf{v}_{0}$ and $Z_{0}$

Add the constraint $\mathbf{w}^{T} \mathbf{v} \geq \gamma Z_{0}$ to $P$

$$
\begin{aligned}
& \text { for } i=1 \text { to } n \\
& \text { Let } c_{i}=1 \text { and } c_{j}=0, \forall j \neq i \\
& \text { Maximize } P \text {, starting from } \boldsymbol{v}_{i-1} \\
& \text { to get } \mathbf{v}_{i} \text { and } Z_{i} \\
& \operatorname{maxFlux}_{i}=Z_{i}
\end{aligned}
$$

Once all maximization problems have been solved, the minimization problems are solved in the same way, starting from $\mathbf{v}_{0}=\mathbf{v}_{n}$.

An important difference between the various LP solvers available is their ability to exploit multiple core CPUs or multi-processor CPUs to increase performance. The GLPK solver, for example, is a single threaded application. When running on a quad-core machine with hyperthreading enabled, the CPU load is only at $12-13 \%$. On multi-core machines, a significant speedup can often be achieved by simply running multiple instances of fastFVA, each working on a different subset of the $n$ reactions.

The fastFVA package runs within the Matlab environment, which will facilitate the use of fastFVA by users less experienced in programming. In addition, many biochemical network models can be imported into Matlab using the Systems Biology Markup Language (SBML) and the COBRA toolbox [10].

The fastFVA code is written in $\mathrm{C}++$ and is compiled as a Matlab EXecutable (MEX) file (additional file 1). Matlab's PARFOR command is used to exploit multi-
Table 1 The models used in the experiments

\begin{tabular}{lccc}
\hline Model & References & Reactions & Metabolites \\
\hline T. maritima & {$[13]$} & 647 & 565 \\
P. putida & {$[14]$} & 1060 & 911 \\
E. coli & {$[15]$} & 2382 & 1668 \\
Human & {$[16]$} & 3820 & 2785 \\
E-matrix & {$[17]$} & 13694 & 11991 \\
Ecoupled-matrix & {$[5]$} & 13726 & 13047 \\
\hline
\end{tabular}

core machines. Two different solvers are supported, the open-source GLPK package [9], and the industrial strength CPLEX solver from IBM.

\section{Results and Discussion}

We evaluated the performance of fastFVA on six biochemical network models ranging from approx. 650 up to 13,700 reactions (Table 1 , additional file 2).

\section{Performance evaluation}

Four metabolic networks and two versions of a genomescale network of the transcriptional and translational ( $\operatorname{tr} / \mathrm{tr}$ ) machinery of E. coli were used for testing the fastFVA code (Table 1). The biomass reaction was used as an objective in the metabolic models, while the demand of ribosomal $50 \mathrm{~S}$ subunit was used as the objective in the $\mathrm{tr} / \mathrm{tr}$ models. In all cases, flux distributions corresponding to at least $90 \%$ of optimal network functionality were sought.

The fastFVA code was tested on a DELL T1500 desktop computer with a $2.8 \mathrm{GHz}$ quad core Intel i7 860 processor with hyperthreading enabled and Windows 7.

\section{Running times}

The running times are given in Table 2 where fastFVA is compared to the direct implementation of FVA found in the COBRA toolbox [10]. The observed speedup is significant, ranging from 30 to 220 times faster for GLPK and from 20 to 120 times faster for CPLEX. The minimum and maximum flux values obtained with fastFVA were essentially identical to the values obtained with the direct approach (data not shown).

Table 2 Running time (s) for fastFVA versus a direct FVA implementation

\begin{tabular}{lcccc}
\hline & \multicolumn{2}{c}{ GLPK } & \multicolumn{2}{c}{ CPLEX } \\
& FVA & fastFVA & FVA & fastFVA \\
\hline T. maritima & 10.3 & 0.3 & 4.3 & 0.2 \\
P. putida & 37.0 & 1.1 & 12.3 & 0.3 \\
E. coli & 340.0 & 2.5 & 119.5 & 1.5 \\
Human & 2217.8 & 12.5 & 659.8 & 5.4 \\
E-matrix & 12263.1 & 184.0 & 9514.6 & 108.1 \\
Ecoupled-matrix & $>120 \mathrm{~h}$ & 1919.4 & 30630.1 & 1421.7 \\
\hline
\end{tabular}




\section{Other uses of fastFVA}

The fastFVA code can be used to compute the fluxspectrum [11], a variant of metabolic flux analysis, simply by setting $\gamma=0$ in (2). The $\alpha$-spectrum [12], which has been used to study flux distributions in terms of extreme pathways, can also be computed with fastFVA. In this case, the parameter $\gamma$ in (2) is set to zero and the $\mathbf{S}$ matrix is replaced by a matrix $\mathbf{P}$ containing the extreme pathways as its columns.

\section{Conclusions}

With this efficient FVA tool in hand, new questions can be addressed to study the flexibility of biochemical reaction networks in different environmental and genetic conditions. It is now possible to design computational experiments requiring hundreds or even thousands of FVAs.

\section{Availability and requirements}

The fastFVA package is freely available at http://notendur.hi.is/ithiele/software/fastfva.html together with precompiled binaries for Linux and Microsoft Windows. The fastFVA code runs under Matlab and relies on third-party solvers to solve linear optimization problems. Two such solvers are supported, the open source GLPK [9] and the industrial strength CPLEX (IBM Inc.) The fastFVA code is written in $\mathrm{C}++$ and is compiled as a Matlab EXecutable function (MEX). It is released under GNU LGPL.

\section{Additional material}

Additional file 1: This file contains the $\mathrm{C}++$ source code, the precompiled binaries, an example on how to use fastFVA and scripts for carrying out the experiments described above.

Additional file 2: This file contains the six metabolic networks used in the experiments

\section{Acknowledgements}

We want to thank the authors of GLPK, GLPKMEX and CPLEXINT for making their code publicly available. We would like to thank the anonymous reviewers for their helpful comments. The authors are also grateful to Ronan M.T. Fleming for valuable discussions.

This study was supported by the Office of Science (ASCR), Department of Energy, under Award Number DE-SC00092009 ("Numerical Optimization Algorithms and Software for Systems Biology").

\section{Author details}

${ }^{1}$ Center for Systems Biology, University of Iceland, Reykjavik, Iceland. ${ }^{2}$ Faculty of Industrial Engineering, Mechanical \& Industrial Engineering \& Computer Science, University of Iceland, Reykjavik, Iceland.

\section{Authors' contributions}

IT conceived and designed the study. SG implemented fastFVA and carried out the experiments. Both authors wrote the manuscript and approved its final version.
Received: 26 May 2010 Accepted: 29 September 2010

Published: 29 September 2010

\section{References}

1. Fell D, Small J: Fat synthesis in adipose tissue. An examination of stoichiometric constraints. Biochem J 1986, 238(3):781.

2. Savinell J, Palsson B: Network analysis of intermediary metabolism using linear optimization. I. Development of mathematical formalism. J Theor Biol 1992, 154(4):421-454

3. Mahadevan $\mathrm{R}$, Schilling $\mathrm{C}$ : The effects of alternate optimal solutions in constraint-based genome-scale metabolic models. Metabolic engineering 2003, 5(4):264-276.

4. Reed J, Palsson B: Genome-scale in silico models of $E$. coli have multiple equivalent phenotypic states: assessment of correlated reaction subsets that comprise network states. Genome Research 2004, 14(9):1797.

5. Thiele I, Fleming R, Bordbar A, Schellenberger J, Palsson BO: Functional characterization of alternate optimal solutions of Escherichia coli's transcriptional and translational machinery. Biophysical Journal 2010.

6. Bushell M, Sequeira S, Khannapho C, Zhao H, Chater K, Butler M, Kierzek A, Avignone-Rossa C: The use of genome scale metabolic flux variability analysis for process feed formulation based on an investigation of the effects of the zwf mutation on antibiotic production in Streptomyces coelicolor. Enzyme and Microbial Technology 2006, 39(6):1347-1353.

7. Pharkya $P$, Maranas C: An optimization framework for identifying reaction activation/inhibition or elimination candidates for overproduction in microbial systems. Metabolic engineering 2006, 8:1-13.

8. Feist A, Zielinski D, Orth J, Schellenberger J, Herrgard M, Palsson B: Modeldriven evaluation of the production potential for growth-coupled products of Escherichia coli. Metabolic Engineering 2009, 12:173-186.

9. Makhorin A: Glpk (gnu linear programming kit), version 4.42.[http://www. gnu.org/software/glpk/].

10. Becker S, Feist A, Mo M, Hannum G, Palsson B, Herrgard M: Quantitative prediction of cellular metabolism with constraint-based models: the COBRA Toolbox. Nat Protoc 2007, 2(3):727-738.

11. Llaneras F, Pico J: An interval approach for dealing with flux distributions and elementary modes activity patterns. Journal of theoretical biology 2007, 246(2):290-308.

12. Wiback S, Mahadevan R, Palsson B: Reconstructing metabolic flux vectors from extreme pathways: defining the $a$-spectrum. Journal of theoretical biology 2003, 224(3):313-324.

13. Zhang Y, Thiele I, Weekes D, Li Z, Jaroszewski L, Ginalski K, Deacon A, Wooley J, Lesley S, Wilson I, Palsson B, Osterman A, Godzik A: ThreeDimensional Structural View of the Central Metabolic Network of Thermotoga maritima. Science 2009, 325(5947):1544.

14. Nogales J, Palsson B, Thiele I: A genome-scale metabolic reconstruction of Pseudomonas putida KT 2440: iJN 746 as a cell factory. BMC Systems Biology 2008, 2:79.

15. Feist A, Henry C, Reed J, Krummenacker M, Joyce A, Karp P, Broadbelt L, Hatzimanikatis $\mathrm{V}$, Palsson B: A genome-scale metabolic reconstruction for Escherichia coli K-12 MG1655 that accounts for 1260 ORFs and thermodynamic information. Molecular systems biology 2007, 3.

16. Duarte N, Becker S, Jamshidi N, Thiele I, Mo M, Vo T, Srivas R, Palsson B: Global reconstruction of the human metabolic network based on genomic and bibliomic data. Proc Natl Acad Sci 2007, 104(6):1777.

17. Thiele I, Jamshidi N, Fleming RMT, Palsson B: Genome-Scale Reconstruction of Escherichia coli's Transcriptional and Translational Machinery: A Knowledge Base, Its Mathematical Formulation, and Its Functional Characterization. PLoS Comput Biol 2009, 5(3):e1000312.

doi:10.1186/1471-2105-11-489

Cite this article as: Gudmundsson and Thiele: Computationally efficient flux variability analysis. BMC Bioinformatics 2010 11:489. 